\title{
Towards a new generation of Charged Lepton Flavour Violation searches at the Paul Scherrer Institut: The MEG upgrade and the Mu3e experiment
}

\author{
Angela Papa ${ }^{1, a}$ \\ ${ }^{1}$ University of Pisa and INFN, 56126 Pisa (Italy) and Paul Scherrer Institut, 5232 Villigen (Switzerland) \\ On behalf of the MEGII and Muße collaborations
}

\begin{abstract}
The MEG experiment has recently set a new upper limit on the branching ratio of the $\mu^{+} \rightarrow \mathrm{e}^{+} \gamma$ decay, $\mathcal{B}\left(\mu^{+} \rightarrow \mathrm{e}^{+} \gamma\right)<4.2 \times 10^{-13}$ (at $90 \%$ confidence level) and un upgrade of the experiment (the MEGII experiment) is ongoing with the aim of improving the single event sensitivity (SES) by one order of magnitude with respect to the previous MEG experiment's SES. The strong scientific motivation associated with the charged Lepton Flavour Violation (cLFV) searches pushes also towards searching for the complementary muon $\operatorname{cLFV~} \mu^{+} \rightarrow \mathrm{e}^{+} \mathrm{e}^{+} \mathrm{e}^{-}$decay with a completely new apparatus, the Mu3e experiment, aiming at a SES improved by at least three orders of magnitude with respect to the previous SINDRUM experiment's SES (Mu3e phase I). An ultimate SES of few $\times 10^{-16}$ is foreseen requiring $10^{9} \mu / \mathrm{s}$ (Mu3e phase II). Both experiments will be hosted at the Paul Scherrer Institut which currently delivers the most intense continuous low energy muon beam in the world up to few $\times 10^{8} \mu / \mathrm{s}$. The status of both the MEGII and Mu3e phase I experiments is given.
\end{abstract}

\section{Introduction}

Lepton flavour violation (LFV) research is currently one of the most exciting branches of particle physics due to its high sensitivity to unveil New Physics (NP) [1]. There are both strong theoretical motivations and experimental evidences which suggest that the Standard Model (SM), the theoretical framework describing the fundamental particles and interactions and recently enriched by the discovery of the Higgs boson, is not the ultimate theory and must be extended [2]. The unknown physics needed to address the left open question of the SM is what is called New Physics.

In the SM lepton flavour violation processes like $\mu^{+} \rightarrow \mathrm{e}^{+} \gamma$ are not allowed. Actually in the SM the lepton flavour is an accidental symmetry, not related to the gauge theory structure and naturally violated in the SM extensions. Furthermore the observation of neutrino oscillations has clearly demonstrated that neutral lepton flavour is not conserved [3-5]. This implies that charged LFV (cLFV) processes, such as $\mu^{+} \rightarrow \mathrm{e}^{+} \gamma$ or $\mu^{+} \rightarrow \mathrm{e}^{+} \mathrm{e}^{+} \mathrm{e}^{-}$decays, can occur in simple extended SM versions which take into account for neutrino oscillations (i.e. including Dirac neutrinos), although strongly suppressed (i.e. $\mathcal{B}\left(\mu^{+} \rightarrow \mathrm{e}^{+} \gamma\right) \approx$ $10^{-54}$ ). On the other hand, Beyond SM (BSM) extensions enhance the predictions for cLFV branching ratios at levels that can be measured by the experiments currently in preparation [6-10]. Due to the fact that at these SES levels there are not contributions coming from SM processes

\footnotetext{
ae-mail: angela.papa@psi.ch;angela.papa@unipi.it
}
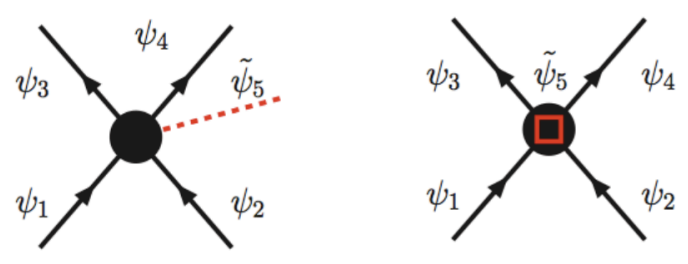

Figure 1. Complementarity between direct (left) and indirect (right) BSM particle searches.

(i.e. SM background free) an evidence of such a decays would be a clear signature of NP (behind neutrino oscillations) and therefore cLFV searches turn out to be ideal probes for NP hints.

The strong complementarity between direct and indirect NP search with accelerator machines is schematically shown in Figure 1. The first case (Figure 1, left) refers to the direct searches that can be performed at energy frontiers where the new particles (red dashed line) would be directly produced in the final state while the second case (Figure 1, right) indicates the indirect searches, typically performed at the precision and intensity frontiers and at which cLFV searches belong to, where the contribution 
of the new particles (red line) would indirectly appear enhancing the probability of processes that otherwise would be strongly suppressed or never occur.

The most general approach to describe the NP under the assumption that the NP characteristic energy scale is well above the energies explored so far is to write an effective lagrangian made by the sum of the SM lagrangian and all the other new terms, suppressed by inverse powers of the new heavy mass scale $\Lambda$ [11-13]:

$$
\mathcal{L}_{e f f}=\mathcal{L}_{\mathcal{S M}}+\sum_{d>4} \frac{c_{n}^{(d)}}{\Lambda^{d-4}} O^{(d)}
$$

where $O$ are the operators, $\mathrm{d}$ is the mass dimension and $c_{n}$ dimensionless coefficients. As it follows from eq. 1 searching for strongly suppressed or forbidden processes offers the unique possibility to probe otherwise unreachable and unexploited new physics energy scale. Following the approach of the effective lagrangian and assuming NP natural coupling the current upper limits on muon cLFV processes translates in new energy scale limits $\Lambda>O(100)$ $\mathrm{TeV}$, independently of the detailed form of the operator responsible for the cLFV process $[14,15]$.

Muonic rare channels such as the $\mu^{+} \rightarrow e^{+} \gamma$ decay, the $\mu^{+} \rightarrow e^{+} e^{+} e^{-}$decay and $\mu^{-} N \rightarrow e^{-} N$ conversion in nuclei are the most promising and complementary cLFV processes (often referred to as "golden muonic channels" [1, 16-20]): (a) The tremendous muon beam intensities (already available: up to few $\times 10^{8} \mu / \mathrm{s}$ (continuous, DC) [21, 22], available soon: $O\left(10^{11}\right) \mu / \mathrm{s}$ (pulsed) $[23,24]$ and understudy: $O\left(10^{10}\right) \mu / \mathrm{s}$ (DC) $[25,26]$, implying for huge statistical samples, together with ultimate performing detectors allow for astonishing muonic cLFV SES; (b) The combined phenomenological analysis of these three processes allow for discriminating the underlying operators generating a potential signal, given different process sensitivities to the different operators. Figure 2 shows the history of cLFV experiments based on the golden muonic channels.

Two of the three golden muonic channels can be studied at PSI which delivers the world's most intense continuous muon beam uniquely suited to study coincidencetype experiments as $\mu^{+} \rightarrow e^{+} \gamma$ and $\mu^{+} \rightarrow e^{+} e^{+} e^{-}$decay searches, where there is more than one particle in the final state.

The MEG experiment searches for the $\mu^{+} \rightarrow e^{+} \gamma$ decay $([27,28])$ and has recently set the most stringent upper limit on its branching ratio $\mathcal{B}\left(\mu^{+} \rightarrow e^{+} \gamma\right)<4.2 \times$ $10^{-13}$ [29-32]. It is a factor 30 improvement over the previous limit set by the MEGA experiment [33] and also the strongest bound on any forbidden decay particle. The strong physics motivation to further explore the $\mu^{+} \rightarrow e^{+} \gamma$ decay has led the collaboration to decide upon an upgrade of the experiment, with the aim to improve the sensitivity by at least one order of magnitude. The MEG upgrade (MEGII) has been approved at PSI and by the Institutions of the international collaboration [34], and is now underway [35].

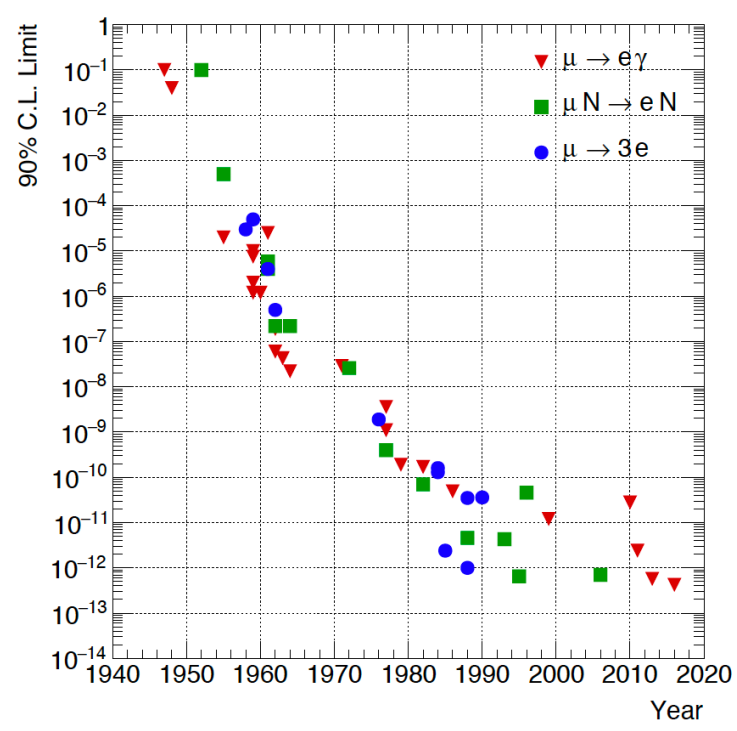

Figure 2. History of cLFV experiments with muons.

Following the mentioned complementary approach the Mu3e experiment at PSI will search for the $\mu^{+} \rightarrow e^{+} e^{+} e^{-}$ decay aiming at a sensitivity of a few $\times 10^{-15}$ [36] (Mu3e phase I) and an ultimate sensitivity of a few $\times 10^{-16}$ (current upper limit $\mathcal{B}\left(\mu^{+} \rightarrow e^{+} e^{+} e^{-}\right)<1.0 \times 10^{-12}$ [37]), and COMET [23] in Japan and Mu2e [24] in US will search for the $\mu^{-} N \rightarrow e^{-} N$ conversion aiming at final sensitivities of few $\times 10^{-17}$ (current upper limit $\mathcal{B}(\mu \mathrm{Au} \rightarrow \mathrm{e} \mathrm{Au}$ ) $\left.<7 \times 10^{-13}[38]\right)$.

\section{The MEGII experiment}

A schematic view of the MEGII apparatus is shown in Figure 3.

In MEGII, surface (positive) muons with a momentum of $29 \mathrm{MeV} / \mathrm{c}$ are stopped in a thin slanted polyethylene target (thickness $140 \mu \mathrm{m}$; angle $15 \mathrm{deg}$ ), located at the center of a magnetic spectrometer.

The signature of a $\mu^{+} \rightarrow \mathrm{e}^{+} \gamma$ decay at rest is a backto-back, mono-energetic, time coincident $\gamma$ and $e^{+}$. The signal event is identified by five observables: The gamma energy $\mathrm{E}_{\gamma}$, the positron energy $\mathrm{E}_{\mathrm{e}}$, the relative gammapositron time $t_{\mathrm{e} \gamma}$, the relative gamma-positron angles $\theta_{\mathrm{e} \gamma}$ and $\phi_{\mathrm{e} \gamma}$. There are two main background sources, the dominant being the accidental coincidences between a high energy positron from the main muon decay $\mu^{+} \rightarrow e^{+} v \bar{v}$ (Michel decay) and a high energy photon from positron annihilation-in-flight or bremsstrahlung or from the radiative muon decay (RMD) $\mu^{+} \rightarrow e^{+} v \bar{v} \gamma$. The other source comes from the RMD itself when neutrinos take off a small amount of energy.

All the $\gamma$ kinematics variables (energy $E_{\gamma}$, time $t_{\gamma}$ and interaction point $X_{\gamma}$ ) are measured using a liquid Xenon (LXe) calorimeter. All the $e^{+}$kinematics variables are measured by a spectrometer made of single cylindrical active drift chamber $\mathrm{CDCH}$ and a highly segmented pixelated Timing Counter pTC mounted inside a gradient mag- 


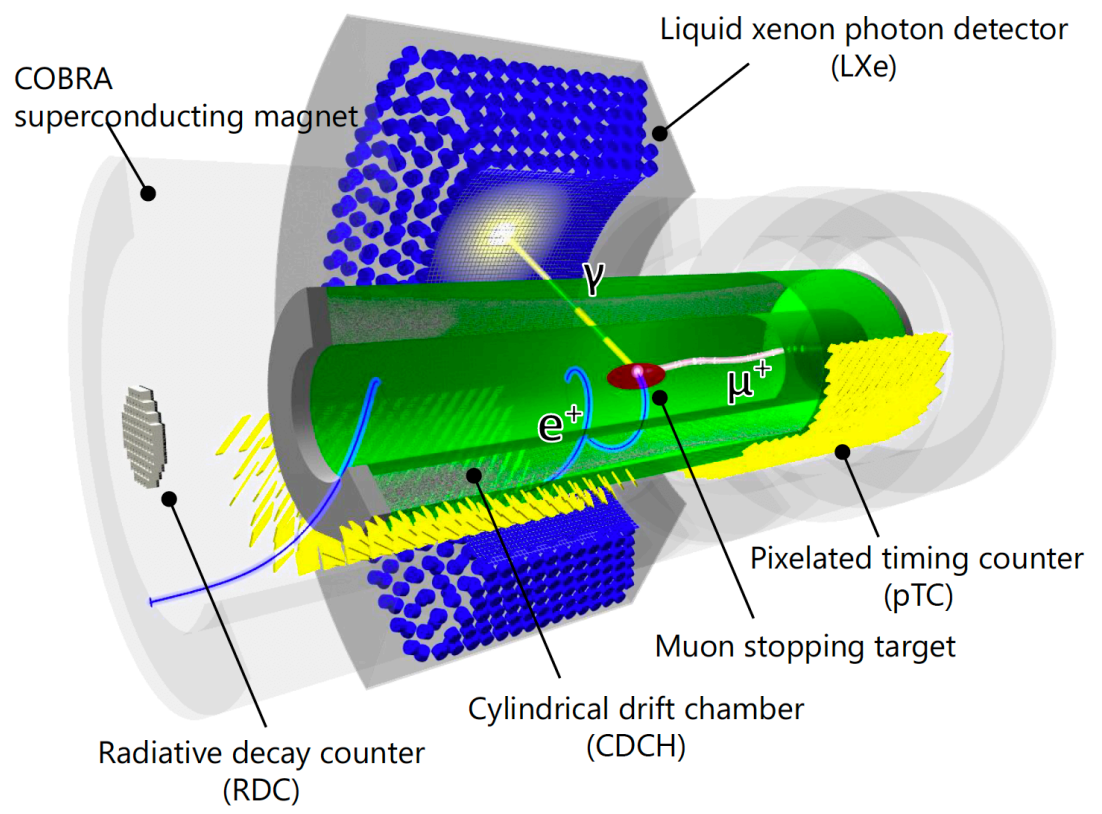

Figure 3. MEGII experimental setup layout (3D view). An example of a $\mu^{+} \rightarrow \mathrm{e}^{+} \gamma$ decay event is shown.

netic field, provided by a COnstant Bending RAdius superconducting magnet (COBRA) with a magnetic field of $1.25 \mathrm{~T}$ at the center. The performance of the experiment is continuously monitored by a variety of calibration methods. All signals are recorded with a custom designed waveform digitizers (DRS), which includes a flexible tigger system to select $\mu^{+} \rightarrow \mathrm{e}^{+} \gamma$ candidate events together with calibration data.

The LXe calorimeter is a "C-shaped" detector made of $\approx 900$ liters of liquid xenon where the photosensors, both photomultipliers and silicon-photomultipliers, are directly immersed. The photosensors have been developed in collaboration with Hamamatsu to work in the VUV region where the xenon scintillation light is emitted $(\approx 175 \pm 5$ $\mathrm{nm}$ ) and at a temperature of $165 \mathrm{~K}$. It is the largest liquid xenon detector in the world based only on scintillation light to preserve the fast Xe scintillating response $\left(\tau_{\alpha}=4.20 \mathrm{~ns}, \tau_{e}=45 \mathrm{~ns}\right)$. The upgrade of the LXe calorimeter involves a denser allocation of photo-detectors on the front face, replacing the current PMTs (Hamamatsu R9869) 2 inch diameter with smaller SiPM (MPPC Hamamatsu VUV sensitive) $12 \times 12 \mathrm{~mm}^{2}$. The better uniformity would imply better energy and position resolutions with a higher impact on the so called shallow events, which are characterized by a less depth conversion point. The reduced amount of material in front of the Xe itself improves the detector performances as well. The $\gamma$ entrance face will be extended along the beam direction by $10 \%$ at each side, increasing also the acceptance. A picture of the inner view of the LXe calorimeter during the Xe filling is given in Figure 4. The comparison between the achieved

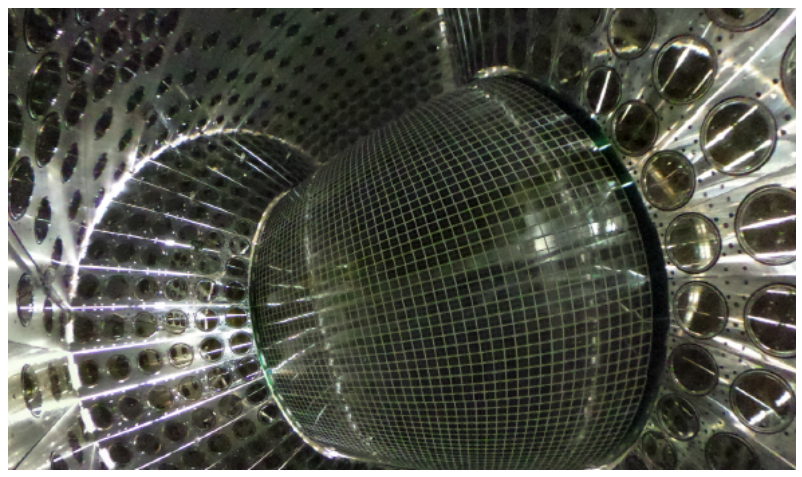

Figure 4. Inner view of the LXe calorimeter. The inner face is assembled with $409612 \times 12 \mathrm{~mm}^{2}$ SiPMs. All the other faces are equipped with the 2 inch. PMT used in MEG.

Table 1. Comparison of the $\gamma$ kinematical variable resolutions (in $\sigma$ ) with the MEG (measured) and MEGII (expected) LXe calorimeter.

\begin{tabular}{lll}
\hline & MEG & MEGII \\
\hline $\mathrm{u}[\mathrm{mm}]$ & 5 & 2.4 \\
$\mathrm{v}[\mathrm{mm}]$ & 5 & 2.2 \\
$\mathrm{w}[\mathrm{mm}]$ & 6 & 3.1 \\
$\mathrm{E}(\mathrm{w}<2 \mathrm{~cm})[\%]$ & 2.4 & 1.1 \\
$\mathrm{E}(\mathrm{w}>2 \mathrm{~cm})[\%]$ & 1.7 & 1.0 \\
$\mathrm{t}[\mathrm{ps}]$ & 67 & 60 \\
\hline
\end{tabular}

MEG and expected MEGII LXe calorimeter performances are summarized in Table 1[39-41].

The new spectrometer has been designed to maintain all the benefits of the former one and to overcome pre- 
vious limitations [42-45]. The main feature is the increased spectrometer granularity which allows to work at higher beam rate and provide better resolutions for all the positron kinematical variables. The positron trajectory is measured up to the point where the positron reaches the new TC tiles, with minimum presence of passive material and an increased number of hits per track, both in the chamber and in the TC. These aspects immediately have an impact on the detector acceptance and efficiency, which is increased by more than a factor 2 , on the positron momentum and angular resolutions and on the positron timing. The new drift chamber will be a $1.91 \mathrm{~m}$ long cylinder with inner and outer radii of $17 \mathrm{~cm}$ and $29 \mathrm{~cm}$ respectively filled with an ultra-low gas mixture of helium and isobutane $\left(\mathrm{C}_{4} \mathrm{H}_{10}\right)$ in the ratio 90:10. The wires will follow a stereo configuration. Each drift cell (with an approximately squared shape, with a variable side size from 6.6 $\mathrm{mm}$ to $9.0 \mathrm{~mm}$ moving from the inner towards the outermost layer) is made of a $20 \mu \mathrm{m}$ diameter gold plated W sense wire surrounded by $40 \mu \mathrm{m}$ diameter silver plated Al field wires in a ratio of 5:1. Two guard wires layers (50 $\mu \mathrm{m}$ diameter silver-plated $\mathrm{Al}$ ) are added at proper radii and voltages for gain equalising of the innermost and outermost layers. The total number of wires would be more than 13000 for an equivalent radiation length per track turn of about $1.6 \times 10^{-3} \mathrm{X}_{0}$. Figure 5 shows the MEGII cylindrical single volume $\mathrm{CDCH}$ before being sealed with a carbon fibre structure.

The new TC is made by a large number of small ultra-fast scintillator plates coupled with SiPM, with improved timing resolution and acceptance. The TC will be made by two sections (Upstream and Downstream) each one made of 256 plates of $\mathrm{BC} 422$ of different size $\left(120 \times 40(50) \times 5 \mathrm{~mm}^{3}\right)$ readout by SiPM $($ AdvanSiD ASD-NUM3S-P-50-High-Gain) (see Figure 6). The high TC segmentation allows to reach a better timing resolution due to multi-hits events (typically one event would be characterised by 7-10 hits), a reduced ambiguity in the positron path length and less scintillation light propagation effects. The use of SiPM overcomes the limited performances of the PMT in magnetic fields, together with a lower transit time spread and higher quantum efficiency [46-49]. The new TC has been successfully tested along the beam line proving that the expected performances at the MEGII conditions have been achieved and confirmed.

A comparison of the performances of the MEG (measured) and MEGII (expected) spectrometer is given in Table 2 .

A large number of calibration and monitoring methods have been introduced and commissioned for the MEG experiment and will be inherited into MEGII with some modifications according to the increased complexity of the upgraded experiment [50-61]. They allow to know in depth the detector behaviour, crucial for building the probability density functions of the observables used to identify a signal event. Furthermore they ensure that the beam characteristics and the detector performances are reached and maintained over the time. Their complexity comes from the fact that some of them require dedicated beams,

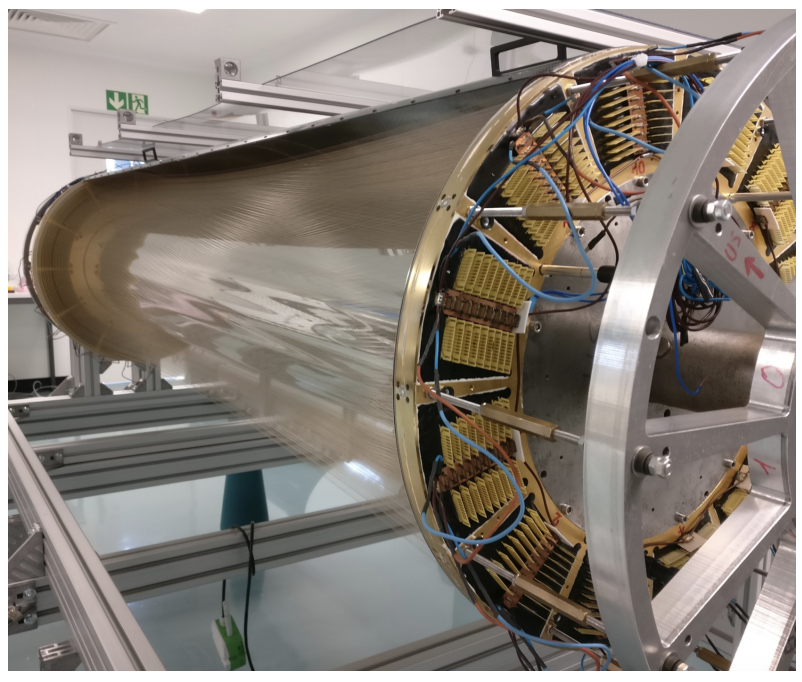

Figure 5. The MEGII single volume $\mathrm{CDCH}$. The hyperbolic profile of the wire envelope is visible.

Table 2. Comparison of the $e^{+}$kinematical variable resolutions (in $\sigma$ ) with the MEG (measured) and MEGII (expected) spectrometer.

\begin{tabular}{lll}
\hline & MEG & MEGII \\
\hline$E_{\text {core }}[\mathrm{keV}]$ & 306 & 130 \\
$\theta_{\text {core }}[\mathrm{mrad}]$ & 9.4 & 5.3 \\
$\phi_{\text {core }}[\mathrm{mrad}]$ & 8.7 & 3.7 \\
$\mathrm{t}$ [ps] & 70 & 35 \\
Tracking efficiency [\%] & 65 & 78 \\
CDCH-pTC matching efficiency [\%] & 45 & 90 \\
\hline
\end{tabular}

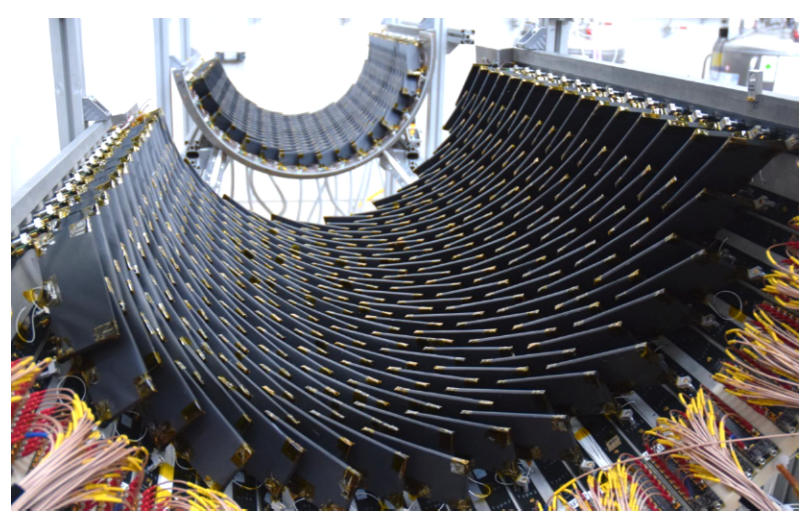

Figure 6. The MEGII pTC. The two sections of the detector (Upstream and Downstream) are shown.

which means different particles (positrons, pions, protons) at different energies with respect to the normal MEG muon beam, dedicated targets, auxiliary detectors up to a devoted $1 \mathrm{MeV}$ Cockcroft-Walton accelerator. Their variety includes the possibility of producing both quasi monochromatic $\gamma$ and $e^{+}$events at the MEG signal region with the unique opportunity to study the detector with real data using signal-like events. The major MEGII calibration and monitoring methods are summarized in Table 3. 
Table 3. The calibration and monitoring methods of the MEGII experiment.

\begin{tabular}{|c|c|c|c|}
\hline Process & Energy & Main Purpose & Frequency \\
\hline Cosmic rays, $\mu^{ \pm}$from atmospheric showers & Wide spectrum $O(\mathrm{GeV})$ & $\begin{array}{l}\text { LXe-DCH relative position } \\
\text { DCH alignment } \\
\text { TC energy and time offset calibration } \\
\text { LXe purity }\end{array}$ & annually \\
\hline Charge exchange, $\pi^{-} \mathrm{p} \rightarrow \pi^{0} \mathrm{n}, \pi^{0} \rightarrow \gamma \gamma$ & $55,83,129 \mathrm{MeV} \gamma$ & LXe energy scale/resolution & annually \\
\hline Radiative $\mu$-decay, $\mu \rightarrow$ eqv & $\begin{array}{l}>40 \mathrm{MeV} \gamma \\
>45 \mathrm{MeV} e^{+}\end{array}$ & $\begin{array}{l}\text { LXe-TC relative timing } \\
\text { Normalisation }\end{array}$ & continuously \\
\hline Normal $\mu$-decay, $\mu \rightarrow e v v$ & $52.83 \mathrm{MeV}$ end-point $e^{+}$ & $\begin{array}{l}\text { DCH energy scale/resolution } \\
\text { DCH and target alignment } \\
\text { Normalisation }\end{array}$ & continuously \\
\hline Mott positrons, $e^{+} \mathrm{X} \rightarrow e^{+} \mathrm{X}$ & $\approx 52.8 \mathrm{MeV} e^{+}$ & $\begin{array}{l}\text { DCH energy scale/resolution } \\
\text { DCH alignment }\end{array}$ & annually \\
\hline Proton accelerator, ${ }^{7} \mathrm{Li}(\mathrm{p}, \gamma){ }^{8} \mathrm{Be}$ & $14.8,17.6 \mathrm{MeV} \gamma$ & LXe uniformity/purity & weekly \\
\hline Proton accelerator, ${ }^{11} \mathrm{~B}(\mathrm{p}, \gamma){ }^{12} \mathrm{C}$ & $4.4,11.6,16.1 \mathrm{MeV} \gamma$ & TC intercalibration/ LXe-TC timing & weekly \\
\hline Neutron generator, ${ }^{58} \mathrm{Ni}(\mathrm{n}, \gamma)^{59} \mathrm{Ni}$ & $9 \mathrm{MeV} \gamma$ & LXe energy scale & weekly \\
\hline Radioactive source, ${ }^{241} \mathrm{Am}(\alpha, \gamma){ }^{237} \mathrm{~Np}$ & $5.5 \mathrm{MeV} \alpha$ & LXe PMT calibration/purity & weekly \\
\hline Radioactive source, ${ }^{9} \mathrm{Be}\left(\alpha_{241} \mathrm{Am}, \mathrm{n}\right){ }^{12} \mathrm{C}^{\star}$ & $4.4 \mathrm{MeV} \gamma$ & LXe energy scale & on demand \\
\hline Radioactive source, ${ }^{57} \mathrm{Co}(\mathrm{EC}, \gamma){ }^{57} \mathrm{Fe}$ & $\mathrm{X}$-rays & LXe-spectrometer alignment & annually \\
\hline LED & VUV region & LXe PMT calibration & continuously \\
\hline Laser & $401 \mathrm{~nm}$ & pTC SiPM calibration & continuously \\
\hline
\end{tabular}

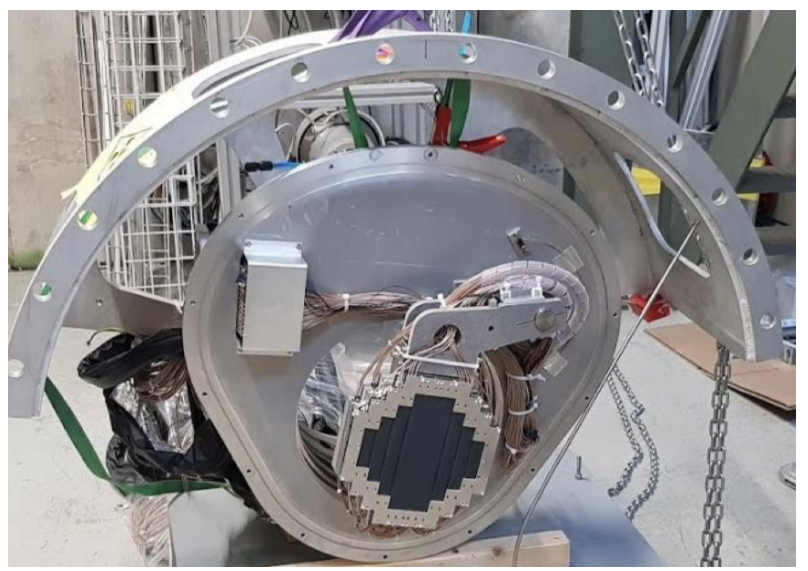

Figure 7. The radiative decay counter fully integrated in the MEGII apparatus.

New auxiliary detectors will be added to the framework of the MEGII experiment, among the others (a) the radiative decay counter RDC, enabling to increase the capability of rejecting the accidental background by tagging low energy positrons associated with the high energy photons in the signal region; (b) a sampling beam monitoring detector based on scintillating fibres coupled to SiPM able to measure both beam profile and rates when needed (Sampling SciFi) [57]. Figure 7 shows the commissioned RDC detector. It is made by $2 \times 2 \times 2 \mathrm{~cm}^{3}$ LYSO crystal coupled to Hamamatsu S12572-025 SiPM and BC418 bars of different width mounted in front of the LYSO crystals coupled to S13360-3050PE. Figure 8 shows the fully tested Sampling SciFi detector made of Multi Cladding Saint Gobain BCF12 $250 \times 250 \mu \mathrm{m}^{2}$ scintillating fibres coupled at both fibre ends to Hamamatsu S13360-3050C SiPM [60].

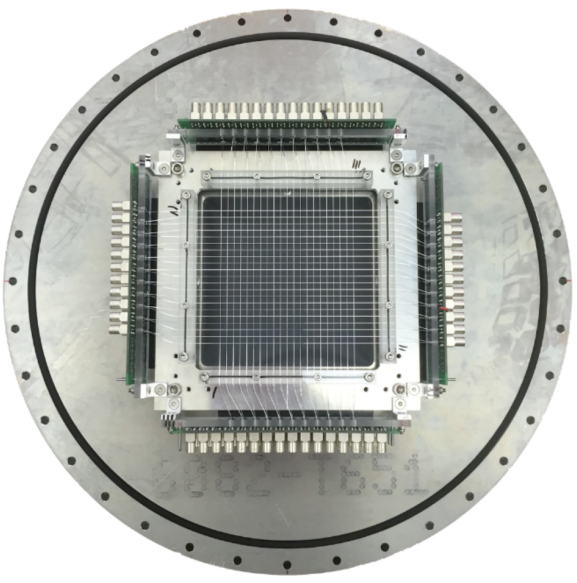

Figure 8. The sampling scintillating fibre detector.

Finally, MEG II requires improvements on the existing DAQ system in terms of an increased number of channels and a higher bandwidth of the waveform digitization system. The new DAQ board (WaveDREAM) has been developed, which combines both the waveform digitizing technology using the DRS4 chip as well as the trigger and splitter functionality of our current system (TDAQ system) [62-68]. More than thousands channels have been tested during the several pre-engineering runs. It included also the trigger electronics and several trigger algorithms, successfully tested and delivered. Figure 9 shows one TDAQ crate, which includes several DAQ boards (total number of channels per crate: 256 channels, 16 channels/boards), 1 trigger board, 1 ancillary board.

In conclusion putting everything together the final MEGII SES is expected to be of $\approx 6 \times 10^{-14}$ for a total experiment lifetime of $O(500)$ DAQ days. 


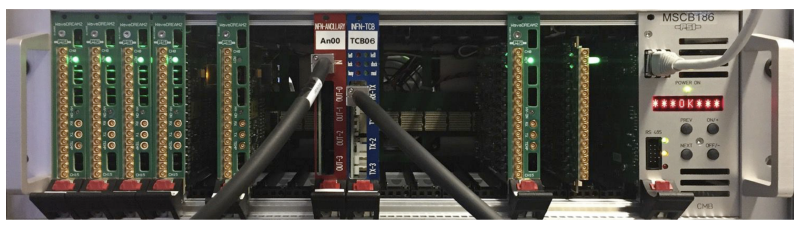

Figure 9. A TDAQ crate equipped with some WaveDREAM boards, a Trigger Concentrator board and an Ancillary board.

\section{The Mu3e experiment}

The Mu3e experiment aims at reaching an ultimate sensitivity of few $\times 10^{-16}$ (or even better) to the branching fraction of the $\mu^{+} \rightarrow e^{+} e^{+} e^{-}$decay [36], four orders of magnitude better than the current limit $\mathcal{B}\left(\mu^{+} \rightarrow e^{+} e^{+} e^{-}\right)<10^{-12}$ set by the SINDRUM experiment [37]. Here we focus on the description of the Mu3e phase I aiming at a SES of few $\times 10^{-15}$.

Positive muons are stopped in a thin double cone target and two positrons and one electron in time coincidence, with a total energy equal to the muon mass and with a null vectorial sum of all the decay particle momenta are searched in the final state as the event signature. There are two many kinds of backgrounds: (a) Overlays of different processes producing three tracks resembling a $\mu^{+} \rightarrow e^{+} e^{+} e^{-}$decay event (accidental background), the most important represented by two Michel positrons one of those undertaking a Bhabha scattering with electrons in the target material, leading to an electron-positron pair from a common vertex; (b) Radiative decays with internal conversion $\mu^{+} \rightarrow e^{+} e^{+} e^{-} v \bar{v}$ with a small energy fraction carried away by the neutrinos: This process is the most serious background for the a $\mu^{+} \rightarrow e^{+} e^{+} e^{-}$decay search.

To be sensitive to the signal and to reject the background excellent momentum resolutions are mandatory to suppress the internal conversion backgrounds and good vertex and timing resolutions are needed to efficiently reduce combinatorial backgrounds. To match those requests the experiment has been design as the following.

The particle momenta will be measured using a novel tracker, made of silicon devices (High-Voltage Monolithic Active Pixel, HV-MAPS [69-71]) for a momentum resolution of about $300 \mathrm{keV} / \mathrm{c}$ over most of the detector acceptance and momentum range, a vertex resolution of $\approx 200 \mu \mathrm{m}$ and a relative fast response $O(10) \mathrm{ns}$. The HV-MAPs have the unique characteristics of integrating the active sensor and the readout in the same device. In current designs, signal amplification and shaping is performed in the pixel itself, whereas the digitisation is carried out in the periphery, a small part at the bottom edge of the sensor. The sensor has digital, zero-suppressed data output via a fast serial link of $1.25 \mathrm{Gbits}^{-1}$. They have pixels with a dimension of $80 \times 80 \mu \mathrm{m}^{2}$, a thickness of 50 $\mu \mathrm{m}$, a time resolution better than $20 \mathrm{~ns}$, a detection efficiency $\epsilon>99 \%$ and a power consumption lower than 350 $\mathrm{mW} / \mathrm{cm}^{2}$. The pixel tracker is made by a central station, made of two inner pixel layers and two outer pixel layers and the re-curl stations, a double copy of the outer pixel layers, displaced upstream and downstream.

The timing is measured precisely by the combination of two detectors: (a) The scintillating fibre hodoscope, a cylindrical time detector device mounted underneath the central outer pixel layers at radius of $6 \mathrm{~cm}$ and with a length of $36 \mathrm{~cm}$. The thinnest available fibres (with a diameter of $250 \mu \mathrm{m}$ ) are arranged to form ribbons of 3-4 fibre layers coupled to SiPM arrays for a timing measurement better than $1 \mathrm{~ns}$ and a full detection efficiency; (b) The scintillating tiles, made by plastic scintillators (EJ228) $6.5 \times 6.5 \times 5 \mathrm{~mm}^{3}$ coupled to SiPMs for a precise timing measurement $(<100 \mathrm{ps})$ at the end of the positron/electron path.

The track and timing information is read out triggerless to a GPU based online filter farm, via three stages of FPGA driven data acquisition boards and an optical link network [72].

All the sub-detectors feature a full detection efficiency (approaching 100\%). They are mounted inside a superconductive magnet which provides a solenoidal field with a default magnetic field of $1 \mathrm{~T}$. Figure 10 shows the schematic view of the experimental set-up.

The preparation of the experiment will follow a staged approach with two major phases. The first phase aims to achieve a SES of $O\left(10^{-15}\right)$ three orders of magnitude improvement in the sensitivity with respect to the previous SINDRUM experiment. The muon beam intensity will increase accordingly, up to exploiting the maximum available beam intensity of few $\times 10^{8}$ muons/s. The second phase aims to reach the ultimate sensitivity of $O\left(10^{-16}\right)$. For this phase a continuous muon stopping rate in the $\mathrm{GHz}$ region is required and feasibility studies are ongoing at PSI to meet also that need (the High Intensity Muon Beam project) $[25,26]$.

The Mu3e collaboration successfully concluded the detector $R \& D$ phase and is starting the prototyping phase. The major results associated with the $R \& D$ are shortly summarized here.

Figure 11 shows a pictures of a telescope assembly based on the MuPix 7 prototype: It is the first small-scale HV-MAP chip which includes all Mu3e functionalities. It has an active area of $2.9 \times 3.2 \mathrm{~mm}^{2}$ with a pixel size of $103 \times 80 \mu \mathrm{m}^{2}$, has been operated up to $1 \mathrm{MHz}$ yielding a full detection efficiency well above $99 \%$ and a timing resolution smaller than $20 \mathrm{~ns}$. These results have been obtained during an extensive test beam campaign exposing the detector at different particles, rates and energies [74]. The first large area prototype, MuPix 8 with an active area of $20 \times 10 \mathrm{~mm}^{2}$ has been then successfully produced and tested as well as shown in Figure 12. MuPix8 is only a factor two less in active area with respect to the final expected chip size needed for the experiment (with an active area of $20 \times 20 \mathrm{~mm}^{2}$ ). MuPix 8 represents the most important milestone towards the production of the final Mu3e chip [75].

Figure 13 shows a scintillating fibre prototype made of four staggered layers of Kuraray SCSF 78 MJ 250 $\mu \mathrm{m}$ diameter fibres readout by SiPM array at both rib- 


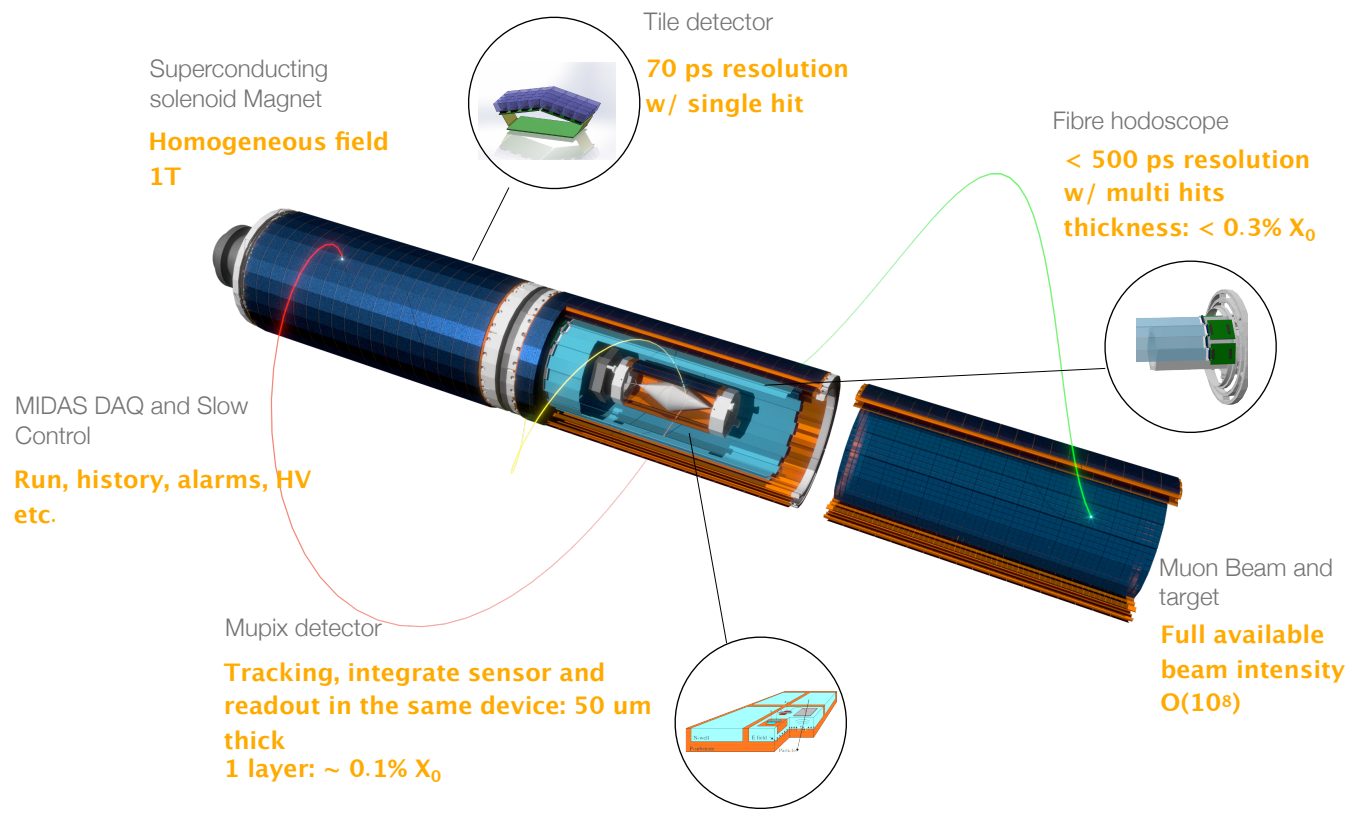

Figure 10. Mu3e experimental setup layout (3D view). An example of a $\mu^{+} \rightarrow \mathrm{e}^{+} \mathrm{e}^{+} \mathrm{e}^{-}$decay event is shown.

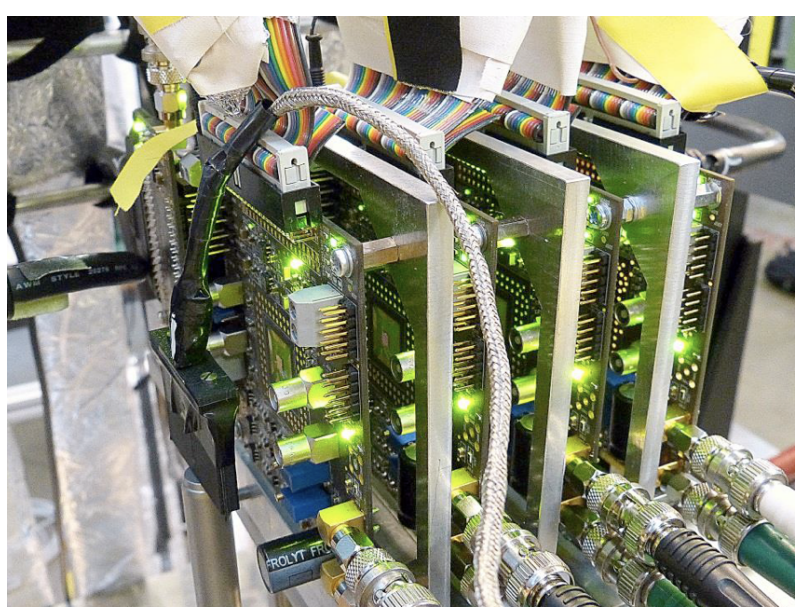

Figure 11. MuPix7: The first small-scale tracker chip prototype which includes all the Mu3e functionalities. The experimental setup used extensively during the 2017-8 test beam campains.

bon ends. The Hamamatsu S13552-HQR SiPM array is shown as well with its flex-print cables. The main challenge of this detector is providing a timing measurement better than $1 \mathrm{~ns}$ with full detection efficiency and with a minimum amount of material $\left(<0.003 X_{0}\right)$. The signals are acquired and digitized with a DAQ acquisition system based on the DRS4 chip at 2 GSample/s [76]. The timing resolution is quoted as the difference between the left and

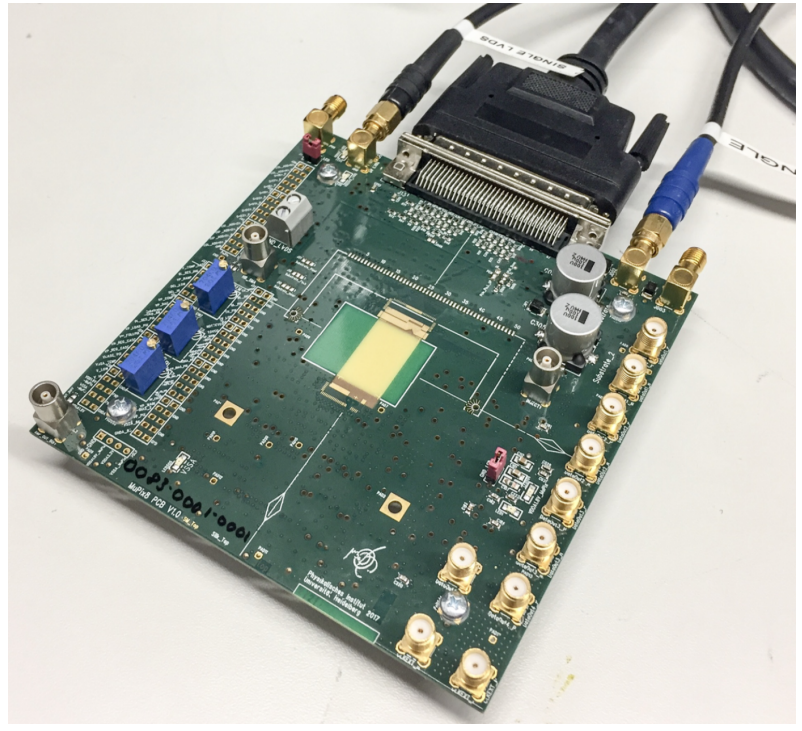

Figure 12. MuPix8: The first large-scale tracker chip prototype with an active area of $20 \times 10 \mathrm{~mm}^{2}$.

right signals divided by a factor of 2 . A timing resolution of $\sigma_{t} \approx 366 \pm 10$ ps has been measured when minimum ionizing particles interact with the detector, with a detection efficiency $\epsilon>96 \%$ [57, 77-79]. The measured timing resolution fulfill the Mu3e needs. 


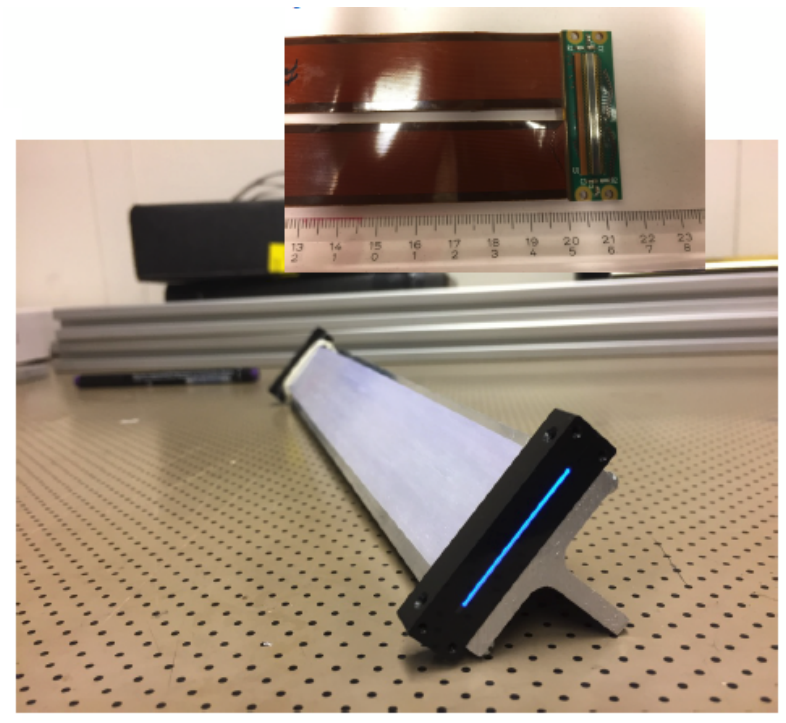

Figure 13. Example of a SciFi ribbon prototype made of four layers of Kuraray SCSF 78 MJ $250 \mu$ m diameter fibres readout by SiPM array. The Hamamatsu S13552-HQR SiPM array is shown as well with its flex-print cables. The temporary Aluminum structure support is also visible.

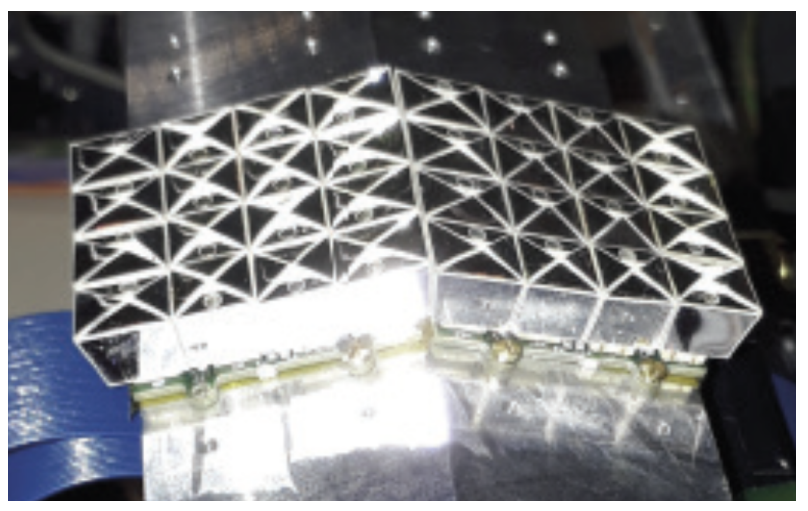

Figure 14. A submodule of the Tile detector. It is made of 32 BC408 $7.5 \times 8.5 \times 5 \mathrm{~mm}^{3}$ elements, each one coupled to Hamamatsu S10362-33-050C SiPM.

Figure 14 shows an example of a single submodule of the Tile detector. Each submodule is made by 32 elements. Each module is made by 14 submodules for a total of 7 modules per re-curl station. The submodule is made of 32 BC408 $7.5 \times 8.5 \times 5 \mathrm{~mm}^{3}$ elements, each one coupled to a silicon photomultiplier Hamamatsu S10362-33-050C and readout with the STiC2 DAQ system [80, 81]. A timing resolution of $O(56)$ ps with full detection efficiency have been measured, fully matching the Mu3e requirements [82]. The final DAQ system for the Fibre and Tile detectors will be based on the muTRiG chip, optimized for the different detector requirements [83].

In conclusion the performances of all sub-detector protoypes measured during the 2017-9 campaign beam test successfully fulfilled the Mu3e demands, proven the suitability of the chosen technologies for the usage in the experiment. The collaboration is currently finalizing the de- tector design and moving to the large scale prototyping phase. This phase will be followed by the preparation for the construction and commissioning of the experiment expected for the 2021 .

\section{References}

[1] Y. Kuno and Y. Okada, Rev. Mod. Phys. 73151 (2001);

[2] J. Ellis, Phil. Trans. R. Soc. A 370, 818 (2012)

[3] Y. Fukuda et al. (Super-Kamiokande Collaboration), Phys. Rev. Lett. 81, 1562 (1998);

[4] Q. R. Ahmad et al. (SNO Collaboration), Phys. Rev. Lett. 87, 071301 (2001);

[5] K. Eguchi et al. (KamLAND Collaboration), Phys. Rev. Lett. 90, 021802 (2003);

[6] S.T. Petcov, Sov. J. Nucl. Phys. 25, 340 (1977);

[7] R. Barbieri and L. Hall Nucl. Phys. B 338, 212 (1994);

[8] R. Barbieri, L. Hall and A. Strumia, Nucl. Phys. B 455, 219 (1995);

[9] J. Hisano, D. Nomura and T. Yanagida, Phys. Lett. B 437, 351 (1998);

[10] M. Raidal et al., Eur. Phys. J. C 57: 13 (2008);

[11] P. Paradisi, Nucl. Phys. B (Proc. Suppl.) 248-250, 8 (2014);

[12] A. Crivellin et al. J. High Ene. Phys. 05117 (2017). https://doi.org/10.1007/JHEP05(2017)117

[13] A. Crivellin et al. arXiv:1611.03409v1 [hep-ph];

[14] J. Albrecht et al., arXiv:1311.5278v2 [hep-ex];

[15] A. de Gouvea and N. Saoulidou, Annu. Rev. Nucl. Part. Sci. 60, 513 (2010).

[16] W. J. Marciano, T. Mori and J. M. Roney, Annu. Rev. Nucl. Part. Sci. 58, 315 (2008);

[17] R. H. Bernstein and P. S. Copper, Phys. Rep. 53227 (2013);

[18] S. Mihara, J.P. Miller, P. Paradisi, and G. Piredda, Annu. Rev. Nucl. Part. Sci. 63, 531 (2013);

[19] L. Calibbi and G. Signorelli, Nuovo Cimento 41, 71 (2018);

[20] G. Cavoto, A. Papa et al., Eur. Phys. J. C 78, 37 (2018);

[21] R. Abela, F. Foroughi, D. Renker The Future of Muon Physics (Springer, Berlin, Heidelberg, New York 1991) 240;

[22] T. Prokscha et al., Nucl. Instr. and Meth. A 595, 317 (2008);

[23] R. Abramishvili et al. (COMET Collaboration), COMET-TDR-2016.pdf

[24] L. Bartoszek et al. (Mu2e Collaboration), https://arxiv.org/pdf/1501.05241.pdf

[25] P. R. Kettle, contribution to Future Muon Sources 2015, University of Hudderseld, United Kingdom; A. Knecht, contribution to NUFACT2017, Uppsala, Sweden; A. Papa, contribution to EPS2019, Ghent, Belgium

[26] F. Berg et al., Phys. Rev. Accel. Beams 19 no.2 024701 (2016); 
[27] A. M. Baldini et al. (MEG Collaboration), MEG Proposal (2002);

[28] J. Adam et al. (MEG Collaboration), Eur. Phys. J. C 732365 (2013);

[29] A. M. Baldini et al. (MEG Collaboration), Eur. Phys. J. C 76434 (2016).

[30] J. Adam et al. (MEG Collaboration), Phy. Rev. Lett. 110, 201801 (2013);

[31] J. Adam et al. (MEG Collaboration), Phy. Rev. Lett. 107, 171801 (2011) ;

[32] J. Adam et al. (MEG Collaboration), Nucl. Phys. B 834, 1 (2010);

[33] M. L. Brooks et al. (MEGA Collaboration), Phys. Rev. Lett. 831521 (1999);

[34] A.M. Baldini et al. (MEGII Collaboration), arXiv:1301.7225v2 [physics.ins-det];

[35] A.M. Baldini et al. (MEGII Collaboration), arXiv:1801.04688 [physics.ins-det];

[36] A. Blondel et al. (Mu3e Collaboration), arXiv:1301.611v1[physics.ins-det];

[37] U. Bellgardt et al. (SINDRUM Collaboration), Nucl.Phys. B 2991 (1988);

[38] W. Bertl et al. (SINDRUM II Collaboration), Eur. Phys. J. C 47337 (2006);

[39] S. Mihara et al., J. Phys. Conf. Ser. 308, 012009 (2011);

[40] W. Ootani et al., PoS(PhotoDet 2012)035;

[41] T. Iwamoto et al., JINST 9, C09037 (2014);

[42] M. Hildebrandt, Nucl. Instr. Meth. Phys. Res. Sect. A 623, 111 (2010);

[43] A. M. Baldini et al., JINST 11, P07011 (2016);

[44] G. Chiarello et al., JINST 12, C03056 (2017);

[45] G. Chiarello et al., Nucl. Instr. Meth. Phys. Res. Sect. A 936, 495 (2019);

[46] M. De Gerone et al., IEEE Trans. Nucl. Sci. 59379 (2012);

[47] M. De Gerone et al., JINST 9 C02035 (2014);

[48] P.W. Cattaneo et al., Nucl. Instr. Meth. Phys. Res. Sect. A 936, 660 (2019);

[49] M. Nishimura et al., Nucl. Instr. Meth. Phys. Res. Sect. A in press, (2020);

[50] A. Papa, IL NUOVO CIMENTO B 122627 (2007);

[51] J. Adam et al. (MEG Collaboration), Nucl. Instr. Meth. Phys. Res., Sect. A 64119 (2011);

[52] A. Baldini et al., Nucl. Instr. Meth. Phys. Res., Sect. A 565589 (2006);

[53] A. Papa et al. Nucl. Phys. B 248-250 (Proc. Suppl.) 121 (2014);

[54] A. Papa et al., JINST 9 C05066 (2014);

[55] A. Papa, G. Cavoto, E. Ripiccini, Nucl. Instr. Meth. Phys. Res., Sect. A 718580 (2013);
[56] A. Papa et al., Nucl. Instr. Meth. Phys. Res., Sect. A 787130 (2015);

[57] A. Papa et al., Nucl. Instr. Meth. Phys. Res., Sect. A 824128 (2016);

[58] G. Rutar et al., Nucl. Instr. Meth. Phys. Res., Sect. A 824575 (2016);

[59] A. Papa and P. Schwendimann, Nucl. Instr. Meth. Phys. Res., Sect. A 936268 (2019);

[60] A. Papa et al., Nucl. Instr. Meth. Phys. Res., Sect. A 936634 (2019);

[61] A. Papa et al., Nucl. Instr. Meth. Phys. Res., Sect. A 93625 (2019);

[62] S. Ritt, R. Dinapoli and U. Hartmann, Nucl. Instr. Meth. Phys. Res., Sect. A 623, 486 (2010);

[63] S.Ritt The WaveDAQ system for the MEGII upgrade contribution to 13th Pisa Meeting on Advanced Detectors (2015);

[64] L. Galli et al., JINST 8, P01008 (2013);

[65] L. Galli et al., JINST 9, P04022 (2014);

[66] M. Francesconi et al., IEEE doi: 10.1109/MOCAST.2016.7495118

[67] L. Galli et al., Nucl. Instr. Meth. Phys. Res. Sect. A 936, 399 (2019);

[68] M. Francesconi et al., Nucl. Instr. Meth. Phys. Res. Sect. A 936, 331 (2019);

[69] I. Peric, Nucl. Instr. Meth. Phys. Res., Sect. A 582 876 (2007);

[70] I. Peric et al., Nucl. Instr. Meth. Phys. Res., Sect. A 731 131 (2013);

[71] N. Berger et al., Nucl. Instr. Meth. Phys. Res., Sect. A 73261 (2013);

[72] S. Bachmann et al., JINST 9, C01011 (2014);

[73] S. Bravar et al., JINST 12, C07011 (2017);

[74] H. Augustin et al., Nucl. Instr. Meth. Phys. Res., Sect. A 845194 (2017);

[75] H. Augustin et al., Nucl. Instr. Meth. Phys. Res., Sect. A 936681 (2019);

[76] S. Ritt, http://www.psi.ch/drs/evaluation-board;

[77] A. Damyanova and A. Bravar, Nucl. Instr. Meth. Phys. Res., Sect. A 845475 (2017);

[78] A. Bravar et al., JINST 12, C07011 (2017);

[79] A. Bravar et al., Nucl. Instr. Meth. Phys. Res., Sect. A in press (2020);

[80] T. Harion et al., JINST 9, C02003 (2014);

[81] T. Harion et al., IEEE doi:10.1109/NSSMIC.2012.6551231;

[82] H. P. Eckert The Mu3e Tile Detector, Thesis dissertation: https : //www.psi.ch/mu3e/ThesesEN/DissertationEckert.pdf

[83] H. Chen et al., JINST 12, C01043 (2017); 